\title{
ANODIC OXTDATION OF DHTYDRONICOTINAMIDE ADENINE DINUCLEOTIDE AT SOLID ELECTRODES; MEDIATION BY SURFACE SPECIES
}

\author{
ZDENEK SAMEC * and PHILIP J. ELVING
}

Deparment of Chemisty. The Unicersity of Michigan, Ann Arbor, MI 48109 (U.S.A.)

(Received 8th October 1981; in revised form 26th August 1982)

\begin{abstract}
The mechanism and kineties for the two-electron oxidation of 1,4-dihydronicotulamide adenine dinucleotide (NADH) to NADt at gold platinum and glassy carbon (GC) rotating cisc electrodes at various solution $\mathrm{pH}$ and NADH concentrations can be rationalized in terms of the electron transfer occurring through mediator redox systems, located close to the electrode surface such as the redox couples formed by nxygen-containing species adsorbed at Au and Pt surfaces. Analogous catalysis of NADH oxidation is provided by sulfide species adsorbed on a gold electrode surface An important factor in differentiating the behavior of the three types of electrodes is the strong adsorption of NADH at Pt and Au, and of $\mathrm{NAD}^{+}$at GC Since oxidation of adsorbed NADH starts at more positive potential than oxidation of bulk NADH, the latter occurs at Au or Pt electrode surfaces covered by adsorbed N.ADH. or at GC electrodes surfaces covered to some extent by adsorbed NAD'. The theory for t, - surface EC catalytic mechanism is considered.
\end{abstract}

\section{INTRODUCTION}

The electrochemical oxidation of 1,4-dihydronicotinamide adenine dinucleotide (NADH) has been studied at glassy carbon (GC), pyrolytic graphite (PG) and platinum (Pt) electrodes from the viewpoints of analysis, role of the NAD ${ }^{+} / \mathrm{NADH}$ redox couple in biological phenomena, and use of the couple in chemical and energy conversion processes (cf. refs. 1-5) *\#. It is well established that NADH undergoes in both aqueous and nonaqueous media an apparently single-step two-electron (2 $e$ ) oxidation [6-9] to enzymatically active $\mathrm{NAD}^{+}[9,10]$ (cf. Fig. 1),

$\mathbf{N A D H} \rightarrow \mathrm{NAD}^{+}+\mathrm{H}^{+}+2 e$

Some evidence has been presented which indicates the involvement of two successive 1 e steps [11], of a slow chemical deprotonation step [12] and of a second order

\footnotetext{
- Permanent address: J. Heyrovsky Institute of Physical Chemistry and Electrochemistry, U tovaren 254. 10260 Prague 10. Crechoslovakia.

- Alhough half-reactions and redax couples may be written or cited as both reductions and oxidations to indicate the direction of movement, potentials cited are consistent with the formulation as reductions. in, Oxtne= Red.
} 
pH-dependent chemical reaction [4]. The latter may be the dimerization or disproportionation of the intermediate radicals $N A H^{+}$and $N A D^{-}[4]$.

Adsorption has been suggested $[8,10,13]$ as a cause for catalytic and inhibition effects observed in the NADH oxidation, but only recently has evidence been presented [14-16] for an adsorption-controlled step. The possible role of oxide formation at GC electrodes has been noted $[8,17]$. The catalytic oxidation of NADH at GC or PG eloctrodes chemically modified by oxygen-containing functionalities has been reported [17,18].

The involvement of adsorption implies the need to control and to monitor the electrode surface carefully. before and during the electrochemical experiment $[4,8]$. The present study examines the correspondence between the rate of NADH oxidation at gold (Au), Pi and GC electrodes, and the state of the electrode surface as characterized electrochemically, as well as the mass-transport and charge-transfer kinetic characteristics of reaction (I).

\section{EXPERIMENTAL}

\section{Chemicals}

NADH, NAD ${ }^{+}$, NMN $^{+}$and ADPR (P-L Biochemicals). NMNH (Sigma), and AMP (Calbiochem) were pure enough to be used as received (cf. Fig. I caption for actonym glassary). Buffer solutions ( $\mathrm{pH} 6.9,8.2,10.5$ ) were $0.25 \mathrm{M}$ in $\mathrm{K}_{2} \mathrm{SO}_{4}$ and $0.05 M$ in phosphate Test solutions were deoxygenated before measurement by nitrogen bubbling.

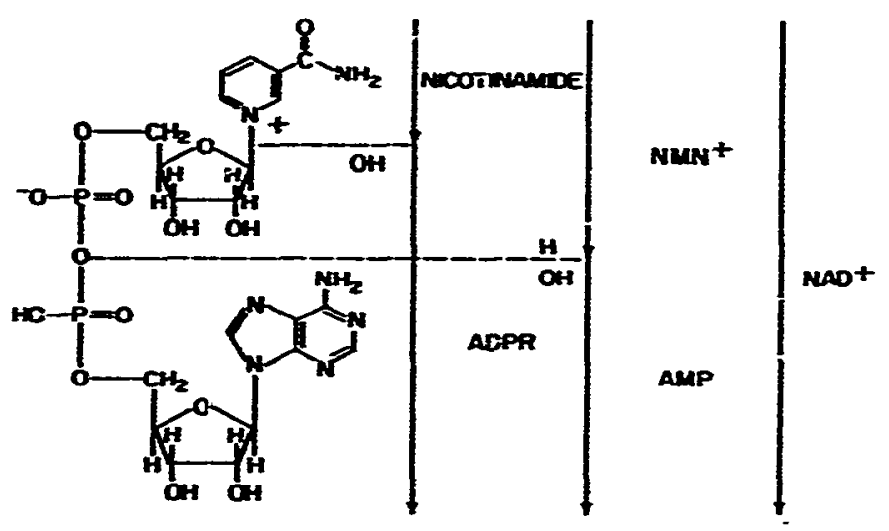

Fig 1. Formula of enymatictïy active $\beta$-nicotinamide adenine dinucleotide (NAD*). Nicotinamide mononveleotode is decignated by $\mathrm{NMN}^{+}$, adenosine diphosphate ribose by $A D P R$ and adenosine $S^{-}$-moaphosplate by AMP. Reduction $\left(2 \in \mathrm{H}^{+}\right.$) of $\mathrm{NAD}^{+}$and $\mathrm{NMN}^{+}$to NADH and NMNH. respectively, resolts in $\mathrm{H}$ addition at $\mathrm{C}(4)$ of the nicoticamide, bond shiff and loss of quaternary character at $\mathbf{N}(1)$ 


\section{Apparatus}

Electrochemical measurements were made with a Princeion Applied Research 170 multipurpose instrument and a three-compartment water-jacketed cell [19]. kept at $25^{\circ}$, whose counter and reference electrode compartments were filled with background solution. A variable speed Caframo motor (1-40 rps) was used to rotate the electrodes.

\section{Electrodes}

Discs of $0.5 \mathrm{~cm}(\mathrm{GC})$ or $0.081 \mathrm{~cm}(\mathrm{Pt} ; \mathrm{Au})$ diameter were cemented into a glass tube (GC) or Teflon cylinder (Au) with an epoxy adhesive. or sealed into a soft glass tube $(\mathrm{Pt})$. The geometric areas, determined by limiting current measurement for hexacyanoferrate(III) reduction at the RDE [20], were $0.169 \mathrm{~cm}^{2}$ for $\mathrm{GC}$ and $5.0 \times 10^{-3} \mathrm{~cm}^{2}$ for Pt and Au. Potentials cited are referred to the saturated calome! reference electrode used. The counter electrode was a gold gauze.

\section{Electrode pretreatment and real surface area}

The electrode surfaces were first polished on rotating 600-mesh SiC paper. In order to obtain a clean reproducible surface as indicated by a steady-state cyclic voltammogram [21] (Fig. 2), the (stationary) electrodes were scanned in the background solution between the onsets of hydrogen and oxygen evolution. e.g., at pH 6.9, between -0.6 and $1.35 \mathrm{~V},-0.44$ and $1.0 \mathrm{~V}$, and -1.6 and $1.0 \mathrm{~V}$ for $\mathrm{Au}$. $\mathrm{Pt}$ and $G C$, respectively; the polarization rate was $0.1 \mathrm{~V} \mathrm{~s}^{-1}$, but $\mathrm{Pt}$ and $\mathrm{Au}$ were first scanned at $10 \mathrm{~V} \mathrm{~s}^{-1}$ for $10 \mathrm{~min}$. This pretreatment was stopped on polarization towards more positive potential at a potential before the onset of oxygen adsorption or surface oxidation. which, at $\mathrm{pH} 6.9$, is $\mathrm{ca}-0.4 .0 .1$ and $0.7 \mathrm{~V}$ for GC. PL. and Au, respectively, (Fig. 2) The start of oxygen adsorption on Au shifts negatively at ca. $-60 \mathrm{mV} / \mathrm{pH}$ and the potential region of oxygen adsorption is broadened [22]: a similar pH effect seen with $\mathrm{Pt}[23-25]$ is connected with $\mathrm{H}^{+}$involvement in the adsorption process.

The actual Au electrode surface area was determined by measuring the charge consumed on oxygen adsorption in $0.5 \mathrm{M} \mathrm{H}_{2} \mathrm{SO}_{4}$ up to the $\mathrm{I}-\mathrm{E}$ curve minimum at $1.45 \mathrm{~V}$ immediately before $\mathrm{O}_{2}$ evolution (Fig. 2); this charge is independent of polarization rate and, based on comparison with BET surface area measurement, corresponds to $400 \mu \mathrm{C} \mathrm{cm}^{-2}$ of real surface area [26]. The area found of $0.042 \mathrm{~cm}^{2}$ yields a roughness factor of $\mathbf{8 . 5}$.

The $\mathrm{Pt}$ electrode area was based on the charge consumed on adsorption of atomic hydrogen in the electrochemical reaction, $\mathrm{H}^{+}+e=\mathrm{H}_{\text {ads. }}$ in $0.5 \mathrm{M} \mathrm{H}_{2} \mathrm{SO}_{2}$ [21]: the generally accepted factor for monolayer and $1: 1 \mathrm{Pt}: \mathrm{H}$ stoichiometry is $210 \mu \mathrm{C}$ $\mathrm{cm}^{-2}$. Evaluation from the voltammogram in $0.5 \mathrm{M} \mathrm{H}_{2} \mathrm{SO}_{4}$ (Fig. 2) [27] gave a real surface area of $0.039 \mathrm{~cm}^{2}$ and a roughness factor of 7.8 .

No standard method is available for determination of the real surface of the GC electrode. 


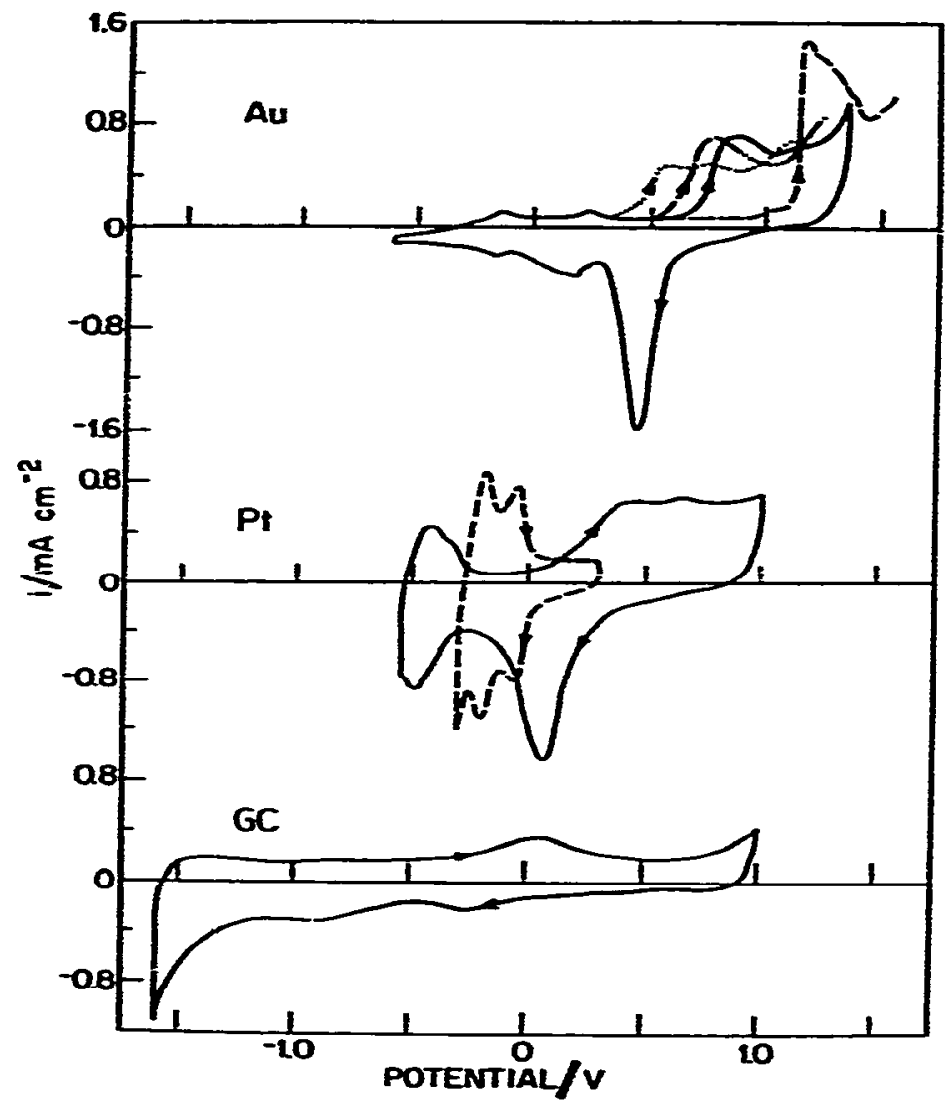

Fig-2. Steady-state voltammograms at stationary $\mathrm{Au}, \mathrm{Pt}$ and $\mathrm{GC}$ electrodes in buffered background electrolyte solutions (0.25 M K $\mathrm{K}_{2} \mathrm{SO}_{4}$; $0.05 \mathrm{M}$ phosphate buffer: pH 6.9 (- - $8.2(-\ldots--)$. 10.5 $(-\cdots,-)$ and in $0.5 \mathrm{M} \mathrm{H}_{2} \mathrm{SO}_{4}(---)$. Rate of polarization: $0.1 \mathrm{Y} \mathrm{s}^{-1}$ : arrowheads indicate the direction of the potential scan. Current density is based on the geometric electrode area.

\section{RESULTS}

\section{Adsorption}

Adsorption at Au and Pt electrodes has been examined by a dip technique.

After its electrochemical pretreatment, the Au electrode was removed from the ceil and was dipped for $5 \mathrm{~min}$ in a $1 \mathrm{mM}$ solution of NADH in the background solution, while rotated at 30 rps. In the blank experiment, the electrode was dipped in the background solution alone. The electrode was then thoroughly washed with water and rodipped in the background solution in the cell. The cyclic voltammogram of a Au electrode after contact with NADH, starting at $-0.6 \mathrm{~V}$ and reversing at 


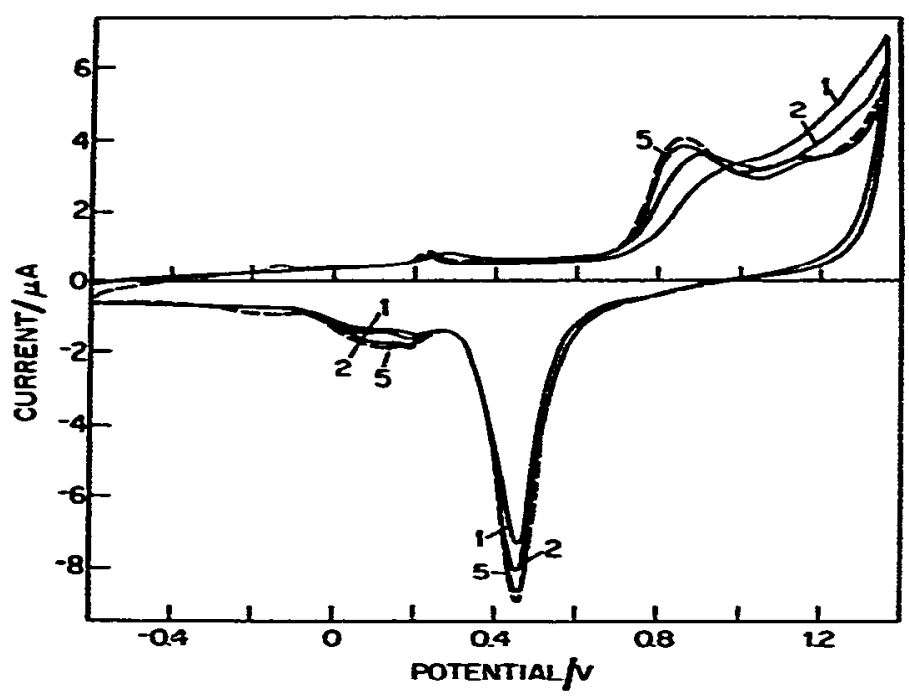

Fig. 3. Cyclic voltammograms at a stationary Au electrode covered by adsorbed NADH and immersed in pH 6.9 solution. Curves are labeled by serial number of repetitive voltage pulses $\left(0.1 \mathrm{~V}^{-1}\right.$ ) between $-0.6 \mathrm{~V}$ (start) and $1.35 \mathrm{~V}$. Dashed curve: steady-state cyclic voltammogram for a clean Au electrode.

$1.35 \mathrm{~V}$ (Fig. 3), is characterized by the inhibition of oxygen adsorption in the 0.7 and $0.9 \mathrm{~V}$ region, increased anodic current between 1.0 and $1.35 \mathrm{~V}$, and the need for at least 10 voltage scans in order to regain the voltammogram of the clean electrode.

The increased anodic carrent positive to $1.0 \mathrm{~V}$ may be due to simultaneous adsorbate oxidation and oxygen adsorption. These two phenomena can be separated by integration of the cyclic voltammogram on the basis that in contrast to adsorbate oxidation. the contribution of oxygen adsorption to charge consumption, $Q^{+}$, on the scan from -0.6 to $1.35 \mathrm{~V}$ is balanced by the adsorbed oxygen reduction contribution to charge consumption, $Q^{-}$, on the return scan. Thus, the charge yield, $Q_{n}=Q_{n}^{+}-Q_{n}^{-}$, at $-0.6 \mathrm{~V}$ on the $n$-th voltage pulse would represent only the charge consumed in adsorbate oxidation. However, integration of the clean electrode steady-state voltammogram yields some oxidation charge, which may correspond to Au dissolution [28], charge lost in the $O_{2}$ evclution, and/or incomplete adsorbed oxygen reduction. Since the possible influence of adsorbed species on Au dissolution and $\mathrm{O}_{2}$ evolution rates is not known, one can only assume that the unbalanced oxidation charge connected with these processes in presence of adsorption is essentially the same as for the clean electrode. Therefore, the charge consumed on adsorbate oxidation is calculated by subtracting $Q_{n}$ for the clean electrode from that for the electrode covered by adsorbate.

In order to isolate the influence, particularly on adsorption, of the component entities in NADH, the following compounds (Fig. 1) were also examined: NAD* , $\mathrm{NMN}^{+}$and NMNH in which the elements of AMP have been removed from 
TABLE I

Characterintion of magniwde of adsorbed layers on gold electrodes

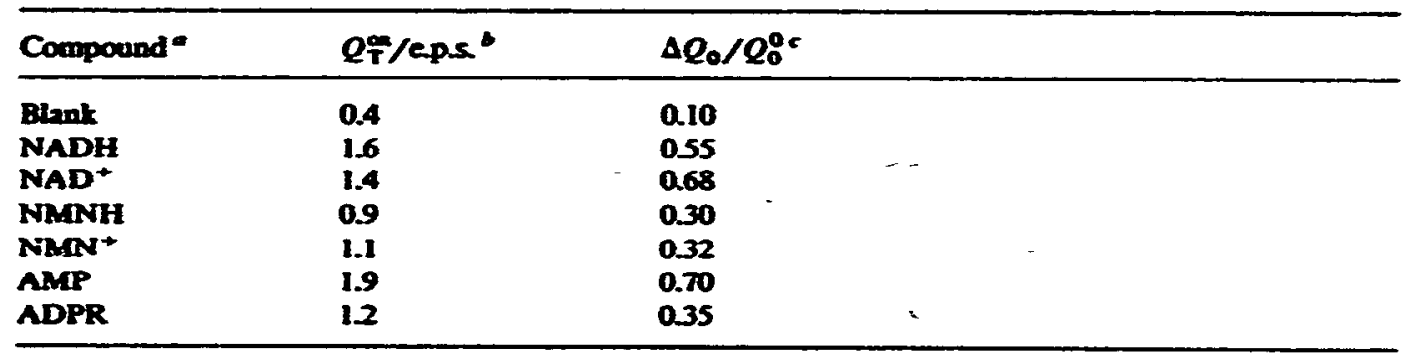

- Presumed adsorbate a fler exposure or electrode to compaund specified.

- Limining tokal oxidation charge $Q \frac{\mathrm{ex}}{3}$ of adsorbed layer. ie. integration of charges for all pulses which are necesary before the steady-state cycic volnmmograms for a clean electrode is obtained (cf. text for melbod of detemination)

- Relative decrease in aygen adsorption $\Delta Q_{0} / Q_{9}^{\circ}$ between 0.7 and 0.9 . which is a relative measure of the exient of adsorption on the electrode Because of the difficulty in measuring the change in surface coverage at Av with adecubete cancentration, it is not clear whether the numbers obtained represent maximum coverage or an intermediate coverage reflecting the differences in energy of adsorption of different compounds adsorbed at the same bull coscentration.

NAD ${ }^{+}$and NADH; AMP; and ADPR in which the nicotinamide moiety has been removed from NAD ${ }^{+}$and NADH. Despite these structural differences, Au electrodes exposed to solutions of NADH, NAD ${ }^{+}$, NMNH, NMN ${ }^{+}$, AMP, or ADPR, exhibit voltammetric behavior qualitatively similar to that described in the two previous paragraphs.

The integrated charge, presumably due only to adsorbate oxidation, approaches a limiting value after 5 scans (NADH, NAD ${ }^{+}$and their components) or one scan (blank experiment), which represents the total charge, $Q_{T}^{\text {ox }}$, necessary for adsorbed layer oxidation (Table 1). The latter can be expressed as the number of electrons per surface gold atom (e.p.s). The experimental factor of $400 \mu \mathrm{C} \mathrm{cm}^{-2}$ for conversion of charge to ep.s. (cf. previous section) is very close to thas expected for an adsorbed monolayer with 1:1 Au:O stoichiometry [21] and thereby can be taken as the equivalent of 2 e.p.s. consumed in a surface charge-transfer reaction.

The extent of adsorption can be characterized by the relative decrease in oxygen adsorption between 0.7 and $0.9 \mathrm{~V}$ on the first scan from -0.6 to $1.35 \mathrm{~V}$ (Fig. 3), i.e, ratio decrease in charge consumed, $Q_{0}^{\circ}$, for the clean electrode (Table 1).

A cyclic voltammogram of a Pt electrode immersed in the background solution afler its contact with $1 \mathrm{mM}$ NADH also exhibits the inhibition of the electrochemical oxygen adsosption and several voltage scans are necessary for complete adsorbed layer axidation. However, measurement of the charge consumed on adsorbed NADH oxidation is not reliable, mainly due to the tendency of Pt to adsorb $\mathrm{O}_{2}$ from the air, to which the electrode is necessarily exposed by the dip technic $Q_{T}^{\text {ox }}$ is roughly twice as large as that for oxidation of NADH adsorbed on Au. 
Because no charge transfer occurs between -0.55 and $0.0 \mathrm{~V}$ at $\mathrm{Pt}$ in the presence of NADH in solution, except for deposition or stripping of adsorbed atnmic hydrogen, the extent of NADH adsorption can be estimated from the decrease in in situ atomic hydrogen adsorption. Surface coverages are 70,73, 78, 79 and $79 \%$ for $0.2 \mathrm{mM}$ and $76 \%$ for $0.4,1.0$ and $1.9 \mathrm{mM}$. Thus, NADH adsorption at Pt increases slightly with bulk NADH concentration.

\section{NADH oxidation at rotating disc electrodes}

Electrode pretreatment in pH 6.9 buffer was stopped at $-0.6(\mathrm{GC}), 0.0(\mathrm{Pt})$ or 0.6 (Au) V; the solution was replaced by one containing NADH. After deoxygenation, a slow $\operatorname{scan}\left(2 \mathrm{mV} \mathrm{s}^{-1}\right)$ towards more positive potential was applied. The wave recorded on both positive and negative polarization was conected for background current (Fig. 4), using for Pt and Au the voltammograms for electrodes covered by adsorbed NADH. When the polarization was stopped in either direction, the NADH oxidation current changed negligibly $(<10 \%)$ during $5 \mathrm{~min}$, indicating that the wave on bcth positive and negative polarization at $\mathrm{Pt}$ and $\mathrm{Au}$ has the stationary character, or, at least, corresponds to the particular electrode surface state, which changes markedly after potentials close to onset of $\mathrm{O}_{2}$ evolution are reached (cf. hysteresis of anodic waves in Fig. 4).

At $2 \mathrm{mM}$ or less NADH, the limiting current, $I_{1}$, for all three electrodes is linearly dependent on $\omega^{1 / 2}$ (angular velocity $\omega=2 \pi f ; f$; $\mathrm{Tps}$ ) and independent of scan direction. Above $2 \mathrm{mM}$, a decline from linearity towards lower values is seen as $f$ approaches 30 rps. At Pt and Au electrodes, $I_{1}$ is constant between pH 6.9 and 10.5 . When $I_{1}$ is related to the geometric electrode area, $A$, all points in Fig. 5 practically

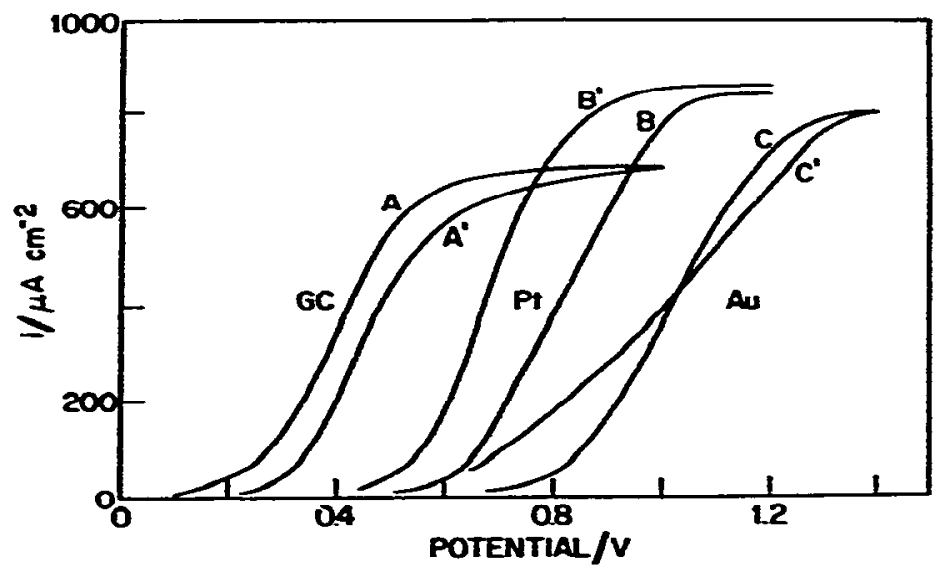

Fig. 4. Amodic wave of NADH at pHi 6.9 corrected for background corrent at GC (A, A2, Pr (B, B) and Au (C,C) rotating disc electrodes, reconded on positive $\left(A, B, C\right.$ ) and negative $\left(A^{\prime}, B^{\prime}, C\right)$ potential scan $\left(2 \mathrm{mV} \mathrm{s}^{-1}\right.$ ) Rotation speed: 30 ms: NADH concentration: $1 \mathrm{mM}$ (GC) and $1.1 \mathrm{mM}$ (Pt. Au). 


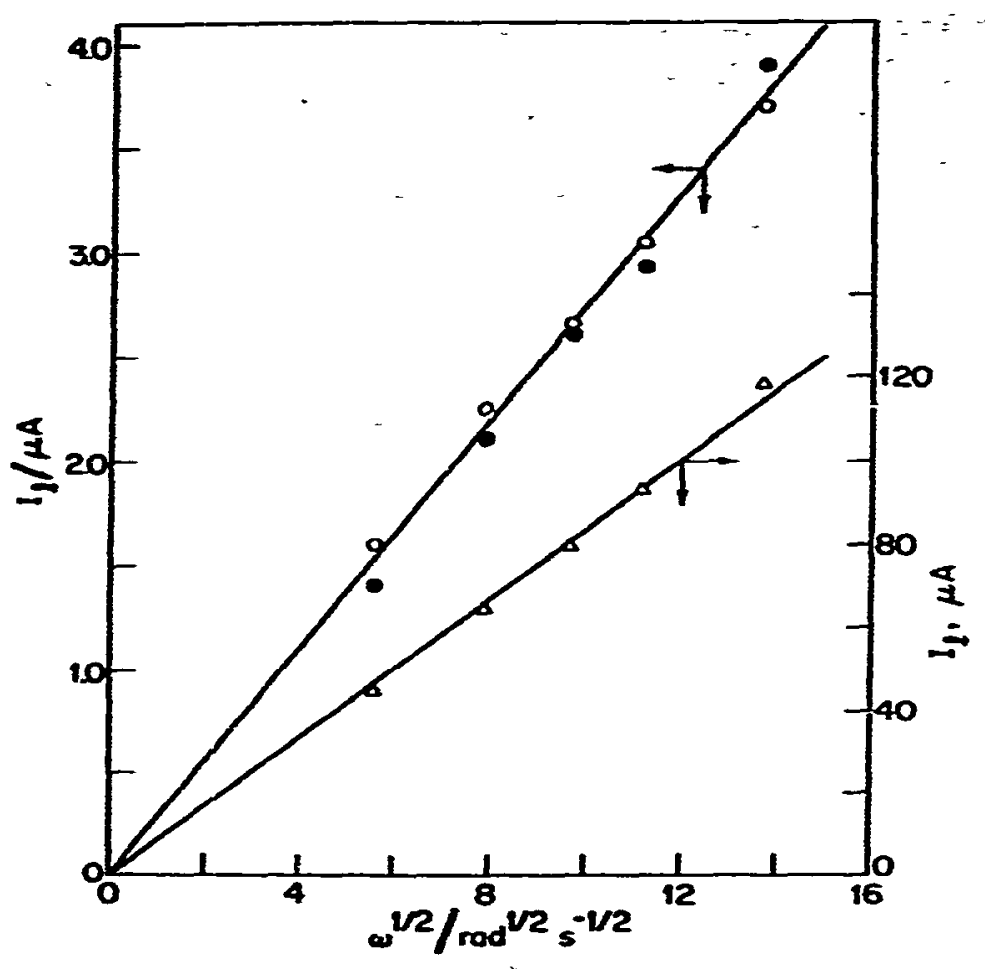

Fis-5. Dependencies of the limiting curreat, $l_{1}$, for the anodic wave of NADH ( $1 \mathrm{mM}$ ) at pH 6.9 an the square root of the angular velocity. $\omega$, for $A v(O), \mathrm{Pl}(0)$ and $G C(\Delta)$ rotaling disc electrodes.

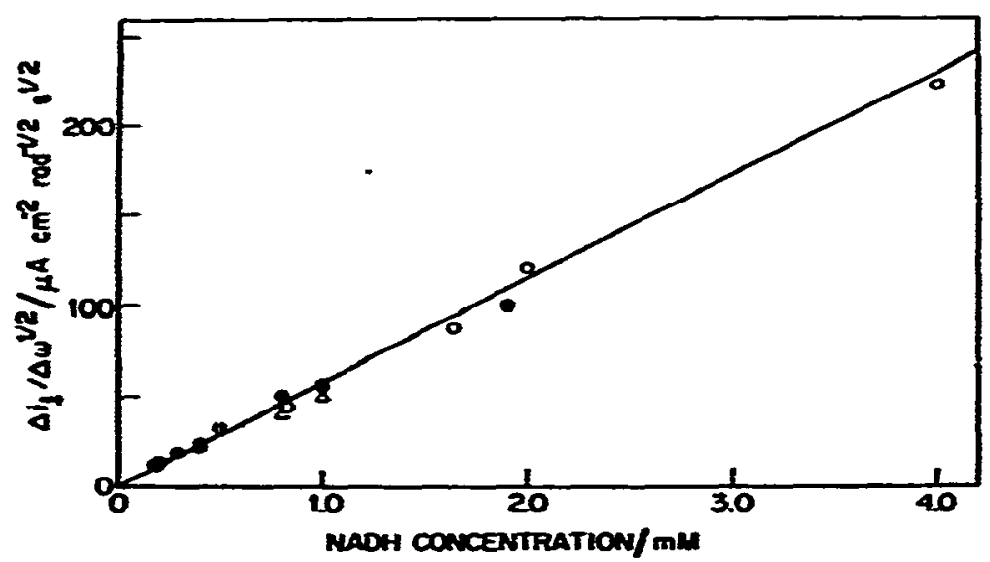

Fis 6. Dependency of the slopes of plots of the fruiting cument density, $i_{1}$, vs square root of the anguiar

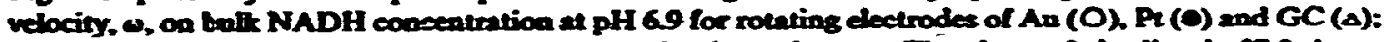

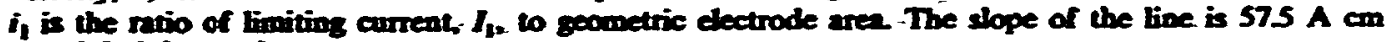
rind $1 / 2$ s $^{1 / 2}$ mall $^{-1}$. 


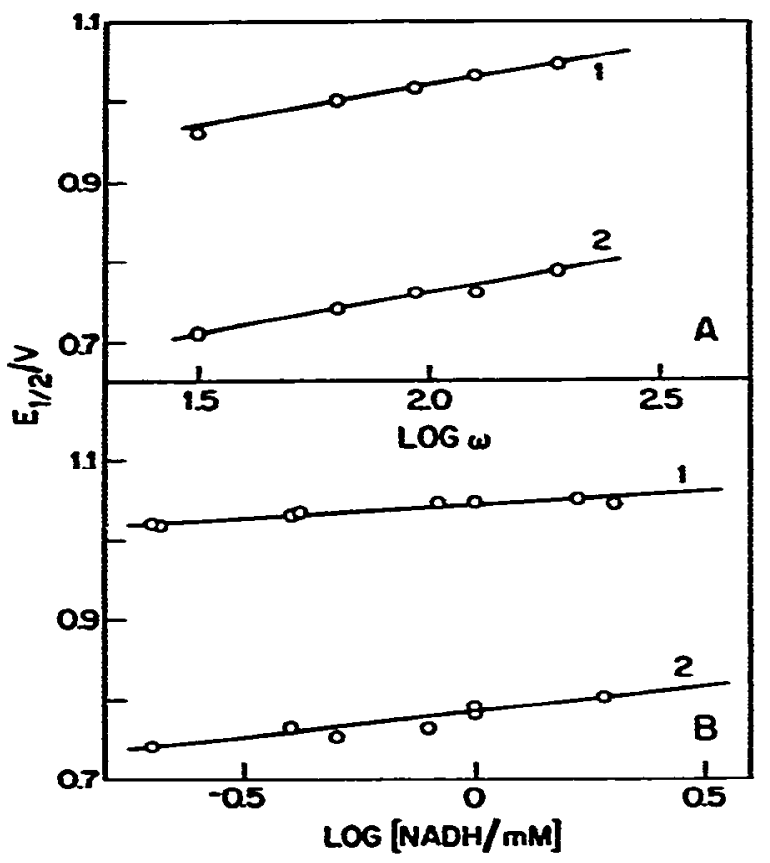

Fig. 7. Dependencies of $E_{1 / 2}$ for NADH at pH 6.9 on (A) electrode rotation speed $\omega$, at $1 \mathrm{mM}$ NADH and (B) bulk NADH concentration at 30 rps. Rotating disc clectrodes: 1 , Au; $2, \mathrm{Pz}$

fall on a single straight line. This is seen in the linear plot of the $I / \omega^{1 / 2}$ ratio, normalized for $A$, vs. NADH concentration (Fig. 6).

With increasing $f$ or NADH concentration, the wave at $P t$ and $A u R D E$ shifts positively (Fig. 7); $\Delta E_{1 / 2} / \Delta(\log \omega)$ is 109 (Au) and $104(\mathrm{Pt}) \mathrm{mV} ; \Delta E_{1 / 2} / \Delta(\log c)$ is 33 (Au) and 66 (Pt) $\mathrm{mV}$. On the other hand, the wave shifts negatively with increasing $\mathrm{pH}$ and the reciprocal slope of the $\log \left[\left(I_{1}-I\right)^{-1}\right]$ vs. $E$ plot decreases (Table 2); $\Delta E_{1 / 2} / \Delta \mathrm{pH}=-53$ (Au) and -28 (Pt) $\mathrm{mV}$.

TABLE 2

Half-wave potentials and log plot slopes * for the NADH axidation wave at mating dice electrodes"

\begin{tabular}{llll}
\hline Electrode & \multicolumn{3}{l}{$E_{1 / 2}($ Slope $) / V$} \\
\\
\cline { 2 - 4 } & pH 6.9 & pH 8.2 & pH 10.5 \\
\hline Au & $1.02(0.19)$ & $0.96(0.19)$ & $0.83(0.11)$ \\
Pt & $0.82(0.16)$ & & $0.72(0.13)$ \\
GC & $0.40(0.17)$ & & \\
\hline
\end{tabular}

- The slopes given in parentheses are the reciprocal slopes in $\mathrm{V}$ for plots of $\log \left(I\left(I_{1}-I\right)^{-1} \mid\right.$ vs. $E$.

- Electrodes were rotnted at $30 \mathrm{mps}$ while being polarized towards more positive potential at $2 \mathrm{mV} \mathrm{s}^{-1}$. 


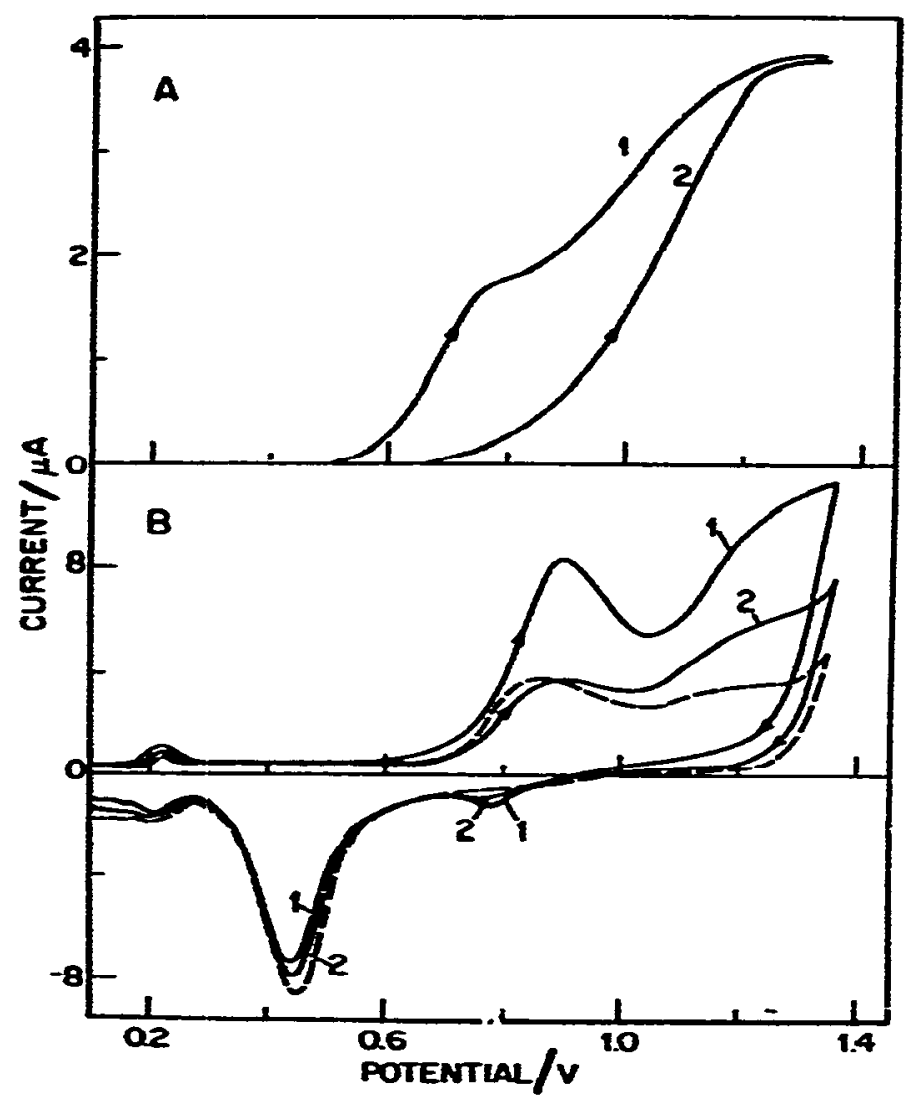

Fig- 8. (A) Anodic wave of NADH (1 mM) at a An rotating disc electrode (30 $\mathrm{mps}$ ) covered by adsorbed sulfide species (poiariation rate $=2 \mathrm{mY} \mathrm{s}^{-1}$ ) and (B) cyclic voltammograms (0.I $\mathrm{V} \mathrm{s} \mathrm{s}^{-1}$ ) at a stationary An electrode covered by adsorbed sulfide species (solid line) or at the clean electrode (dashed line). pH - 6.9. Curves are hbeled by the serial number of the repetitive voltage pulse between (A) 0.4 and $135 \mathrm{~V}$ and (B) -0.6 and $1.35 \mathrm{~V}$. Arrowheads indicate the direction of the potential scan.

\section{Effect of adsorbed sulfide at Au}

Sulfide was adsorbed at a Au surface by the dip procedure described above (dipped for $2 \mathrm{~min}$ in $1 \mathrm{M} \mathrm{Na} \mathrm{N}_{2} \mathrm{~S}$ in $\mathrm{pH} 6.9$ buffer). On cyclic voltammetry of the covered stationary electrode (Fig. 8B), anodic peaks appear at ca. 0.9 and $1.2 \mathrm{~V}$ on the first voltage scan; on subsequent pulses, the more positive peak decreases. At least 10 scans are needed to obtain the clean electrode steady-state voltammogram; $Q_{T}^{\mathrm{ox}}$ on oxidation of the adsorbed sulfide layer is 7.8 ep.s. When the first scan is reversed at $1.0 \mathrm{Y}$ and the range is extended to $1.35 \mathrm{~V}$ on subsequent scans, $Q_{\mathrm{T}}^{\alpha}$ is 2.0 ep.s. for the first scan and 72 eps. for all subsequent, necessary scans. 
The effect of adsorbed sulfide on the NADH oxidation was examined as follows. The anodic wave was recorded as usual (cf. Fig. 4C); the electrode was polarized to $-0.6 \mathrm{~V}$, removed, washed with distilled water, dipped in $! M \mathrm{Na}_{2} \mathrm{~S}$ in base electrolyte for $2 \mathrm{~min}$ while rotated at $30 \mathrm{rps}$, removed, wasned with distilled water. returned to the NADH solution in the cell, and polarized to $-0.6 \mathrm{~V}$; the NADH wave was recorded at $2 \mathrm{mV} \mathrm{s}^{-1}$ towards more positive potential starting at $0.4 \mathrm{~V}$ (Fig. 8A; curve 1). The NADH oxidation is appreciably catalyzed below $0.8 \mathrm{~V}$; at more positive potential, the wave is close to that recorded on Au not covered by sulfide; on repeated scans between 0.4 and $1.35 \mathrm{~V}$, the wave (Fig. 8A; curve 2) practically coincides with that on uncovered $A U$. When the sulfide covered electrode is polarized by repetitive scans between 0.4 and $0.675 \mathrm{~V}$, the catalyzed NADH oxidation proceeds without a change in rate.

Logarithmic analysis of both waves in Fig. 8A indicates that the catalytic effect of adsorbed sulfide is accompanied by a change in the reciprocal of the $\log \left[\left(I_{1}-I\right)^{-1}\right]$ vs. $E$ plot at the wave foot from 190 to $115 \mathrm{mV}$. Repetition of the experiment at 0.2 to $1.0 \mathrm{mM}$ NADH showed no effect on the $\log$ plot slope at the wave foot. However, the latter shifts positively with increasing $N A D H$ concentration: $\Delta E_{1 / 2} / \Delta(\log c)=55$ mV.

\section{DISCUSSION}

\section{Adsorption}

The data indicate that the compounds examined are strongly and irreversibly adsorbed at the gold surface. The compounds lacking the adenine moiety, i.e.. NMNH and $\mathrm{NMN}^{+}$, are adsorbed at Au to a lesser extent than those having that moiety (Table 1). The difference between AMP and ADPR is understandable in terms of the increased number of hydrophilic groups in ADPR. If surface coverage reflects the relative strength of bonding to the surface the adenine moiety is the preferential site for $\mathrm{NADH}$ and $\mathrm{NAD}^{+}$adsorption at $\mathrm{Au}$ and, possibly, Pt. This is in full agreement with the conclusion drawn by Takamura et al. [29]. from their specular reflectivity measurements of adsorption of $\mathrm{NAD}^{+}, \mathrm{NMN}^{+}$, nicotinamide, adenine and adenosine at Au electrode surfaces. Adenine is considered to be the adsorption site for $\mathrm{NAD}^{+}$at $\mathrm{Hg}$ [3] and GC [16] electrodes. The appreciable -dsorption of $\mathrm{NMNH}^{-1}$ and $\mathrm{NMN}^{+}$, however, points to the pyridine moiety possibly being also involved in adsorption at $\mathrm{Au}$.

Inspection of the space-filling model [30] of NADH shows that the estimated minimum projected area for the folded molecule to planes perpendicular or parallel to that of the adenine and pyridine rings is ca. $0.85 \mathrm{~nm}^{2}$ or $1.25 \mathrm{~nm}^{2}$, respectively: the minimum projected area for the flat open moleculs is $1.90 \mathrm{~nm}^{2}$. Since the area per one surface atom of polycrystalline gold is much less, i.e., $0.087 \mathbf{n m}^{2}$, it is probable that more than one surface atom is blocked by a single adsorbed NADH molecule Consequently, uniess NADH is adsorbed in a multilayer, the oxidation charge $Q_{T}^{\text {ax }}$ of 1.6 e.p.s. certainly corresponds to more than 2 electrons consumed in 
the oxidation of one adsorbed NADH molecule The same should hold for the other adsorbates listed in Table 1.

While data indicate that NADH is strongly adsorbed at a $\mathrm{Pl}$ or Au electrode surface it is, in contrast, only weakly adsorbed at GC as compared with the strong adsorption of $\mathrm{NAD}^{+}$at GC from solution or in the electrochemical step [14-16],

$\mathrm{NADH} \rightarrow \mathrm{NAD}_{\mathrm{d}}^{+}+\mathrm{H}^{+}+2 e$

The effect of successive voltage scan oxidation of the adsorbed layer at $\mathrm{Pt}$ and $\mathrm{Au}$ parallels that for $\mathrm{NAD}^{+}$adsorption at GC (eqn. (ID) [14-16], the only difference being that in the former case the adsorbed layer is gradually removed by repetitive scanning while in the latter case it is gradually formed. In general, the behavior of the adsorbed layer at $\mathrm{Pt}$ and $\mathrm{Au}$ can be accounted for on the basis that, as oxidation of adsorbed species occurs close to the onset of $\mathrm{O}_{2}$ evolution, limitation of the available potential range by the latter process may not allow the positive potentials necessary for adsorbate oxidation to be reached in a single voltage scan excursion.

From the high charge consumption on adsorbate oxidation and the small differences in adsorption and oxidation between NADH and NAD ${ }^{+}$, and NMNH and $\mathrm{NMN}^{+}$- we can conclude that $\mathrm{NADH}$ participates in two different oxidation processes at $\mathrm{Au}$ and $\mathrm{Pt}$ electrodes. The first process, described by reaction (I) gives rise to the 2 e anodic wave (Fig. 4); the second can be described by

NADH $\rightarrow(\mathrm{NADH})$ as $\rightarrow \mathrm{X}+n e$

Because oxidation of adsorbed NADH obviously starts at more positive potential than reaction ( $D$, the latter occurs at an electrode surface covered by adsorber NADH, when a An or $P_{1}$ electrode is polarized by a positive potential sweep. At GC, the electrochemical adsorption (reaction (II)) cocurs in place of reaction (III) so that reaction (I) occurs at a surface covered to some extent by adsorbed NAD+.

\section{Mediated mexhanism of NADH oxidation}

Unlike $\mathrm{NAD}^{+}$reduction at a $\mathrm{Hg}_{\mathrm{g}}$ electrode, NADH oxidation at solid electrodes is much less informative about the mechanism of the overall reaction (I). In the former case, the data are best explained by a sequence of two 1 e steps [2],

$\mathbf{N A D}^{+}+e \rightleftharpoons \mathbf{N A D}^{-}$

$\mathrm{NAD}^{-}+\mathrm{H}_{3} \mathrm{O}^{+}+e \rightarrow \mathrm{NADH}+\mathrm{H}_{2} \mathrm{O}$

accompanied by dimerization of the intermediate free radical,

$2 \mathbf{N A D}^{*} \rightarrow(\mathbf{N A D})_{2}$

The rate cosstant for charge-transfer reaction (IV) probably exceeds $1 \mathrm{~cm} \mathrm{~s}^{-1}$ [13]; that for charge-transfer reaction $(V)$ has not yet been evaluated. Since the irreversible polarographic wave corresponding to reaction (V) occurs at potentials as negative as $-1.6 \mathrm{~V}$, reaction $(V)$ is rather slow. The rate constant for dimerization reaction (VI) is of order of $10^{6} \mathrm{M}^{-1} \mathrm{~s}^{-1}$ [2] 
If the sequence of steps (IV) and (V) is reversed on NADH oxidation, reaction (V) can be assumed to be rate-determining. Since the oxidation occurs positive to $0.2 \mathrm{~V}$ (Fig. 4), oxidation of $\mathrm{NAD}^{-}$to $\mathrm{NAD}^{+}$would be sufficiently rapid to outrun the dimerization (reaction (VI)), so that practically no dimer is produced. Under these circumstances, a single anodic 2 e wave should be observed, which corresponds to the irreversible 1 e NADH oxidation (reaction (V)) and which is independent of the nature of electrode material. NADH concentration and solution PII as well. However, since all three effects are involved in NADH oxidation at solid electrodes, modification of the mechanism outlined is required. The effect of electrode material is obviously most significant and it may underlie the effects of both NADH concentration and solution $\mathrm{pH}$.

The correspondence between the rate of NADH oxidation at solid electrodes and the state of the electrode surface can be reasonably explained on the basis of intimate involvement of surface oxygen species in the rate-determining step of overall reaction (I), i.e,

$\mathrm{NADH}+\mathrm{H}_{2} \mathrm{O} \rightarrow \mathrm{NAD}^{-}+\mathrm{H}_{3} \mathrm{O}^{+}+e$

Such correspondence was first suggested by Blaedel and Jenkins [8].

The possible involvement of two surface oxygen redox systems in NADH oxidation is schematically depicted in Fig. 9. The electron-transfer path involves electron exchange between energy levels located at the surface oxygen atom and in the electrode, coupled with electron exchange between energy levels of the surface oxygen atom and the solution species, i.e., NADH molecule. The proton transfer path involves transfer of the proton bound to $C(4)$ of NADH to a third species, which is the proton acceptor, e.g., $\mathrm{H}_{2} \mathrm{O}$, with possible intermediate formation of a bond to the surface oxygen atom:

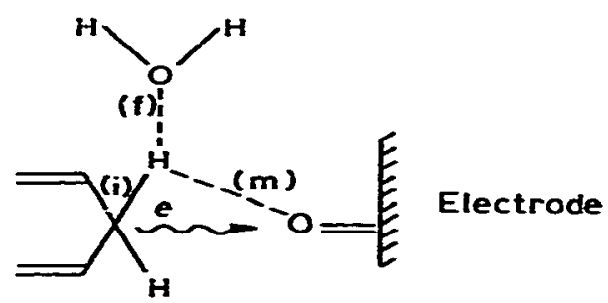

where i, $f$ and $m$ designate the initial, final and possible intermediate bonds, respectively, of the transferred proton.

Thus, two mechanisms can be envisaged for the proton transfer path. In one, electron transfer through the intermediate state located close to the surface oxygen atom and proton transfer are decoupled from each other, ie, the proton bound to C(4) of NADH is not involved in the activated complex formed before electron transfer may occur. The stable intermediate state in the electron-transfer path, which may be but need not be actually formed, would be represented by NAD'H ${ }^{*}$ and $\mathrm{O}_{\mathrm{ad}}\left(\mathrm{OH}_{\text {ads }}\right)$. Subsequently, the proton is transferred from $\mathrm{NAD}^{-} \mathrm{H}^{+}$to a proton acceptor in a distinct chemical step, as supported by previous results [12] 
In the second mechanism, a bond is formed between the transferred proton and the surface atoms, and the intermediate state in the electron-transfer path would be represented by $\mathrm{NAD}^{*}$ and $\mathrm{OH}_{-\mathrm{w}_{6}}\left(\mathrm{H}_{2} \mathrm{O}\right)$. In order that the "inner-bridge" activated complex be formed, reorganization of the proton configuration with respect to both C(4) and surface oxygen atoms and proton acceptor is necessary-

On the whole irrespective of whether or not a stable intermediate state is actually formed, electron transfer through a mediator-energy level may represent a path which is linetically more favorable than direct electron transfer.

The hypothesis that oxidation of NADH may proceed through different mediator redox systems located close tc the electrode surface is further supported by the catalysis of NADH oxidation by surface-attached quinones [17] and by sulfide adsorbed at a Au electrode.

a

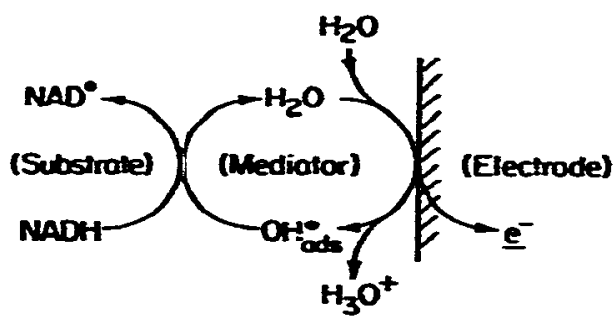

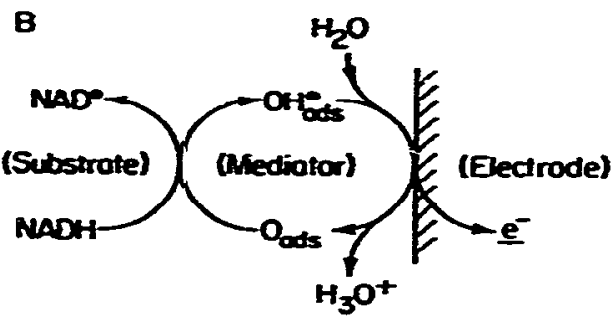

Fig. 9. Sehemicic depictions of possible surface oxygen redox systems as modiators in the oxidation of

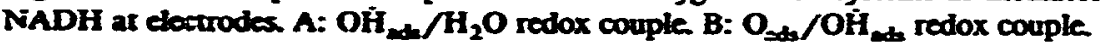

At pH 6.9, sulfide species adsorbed on $A u$ are probably $\mathrm{H}_{2} \mathrm{~S}$ and $\mathrm{HS}^{-}$(pK $_{a}$ for $\mathrm{H}_{2} \mathrm{~S}: 7.04,11.96$ ). The charge of 7.8 e.p.s. consumed on total oxidation of the adsorbed sulfur layer indicates oxidation to the highest normal oxidation stage. Oxidation of the layer "by parts" reveals that the anodic peak at ca. $0.9 \mathrm{~V}$ (Fig. 8E) is due to 2 e oxidation to $S^{(0)}$ and that the peak at ca. $1.2 \mathrm{~V}$ is due to further oxidation to $\mathrm{SO}_{4}^{\mathrm{Z}-}$ (cf. ref. 31).

Significant catalysis of NADH oxidation at a Au electrode covered by adsorbed sulfur species (Fig. 8A) is associated exclusively with species in the lowest oxidation

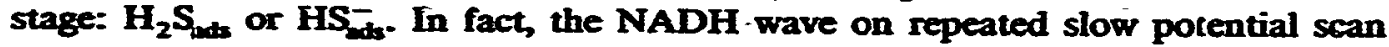


between 0.4 and $1.35 \mathrm{~V}$ (Fig. 8A; curve 2) practically coincides with that at a Au electrode not covered by sulfur species, although $S^{(0)}$ species are still present on the surface (Fig 8B; curve 2). Furthermore, if a Au electrode covered by $\mathrm{H}_{2} \mathrm{~S}_{\mathrm{ds}}$ or $\mathrm{HS}_{\text {ads }}^{-}$ is not polarized more positive than $0.68 \mathrm{~V}$, at which oxidation of these species starts. the catalyzed NADH oxidation proceeds without significant time decay.

Such catalysis can be interpreted in terms of the involvement of such surface redox systems as $\mathrm{HS}_{\text {ida }}^{-} / \mathrm{HS}_{\text {eds }}^{-}$or $\mathrm{HS}_{\text {idd }}^{-} / \mathrm{H}_{2} \mathrm{~S}_{\text {dds }}$ in the electron transfer from NADH to electrode in the same way as such surface redox systems as $\mathrm{O}_{\text {add }} / \mathrm{OH}^{*}$ ads. As the formal potentials of the $\mathbf{H S}_{\text {ids }}^{-} / \mathrm{H}_{2} \mathbf{S}_{\text {eds }}$ and $\mathbf{H S}_{2 \mathrm{ds}}^{-} / \mathbf{H S}_{2 \mathrm{ds}}^{-}$systems are probably less positive than that of the $\mathrm{O}_{\text {add }} / \mathrm{OH}_{\text {eds }}^{-}$system, the oxidation of $\mathrm{NADH}$ is easier when the former systems are involved.

\section{Theory for mediated mechanism}

With the reference to Fig. 9, the surface EC catalytic mechanism will be considered,

$\mathrm{Q}_{\text {ads }}+\mathrm{H}^{+}+e \rightleftarrows \mathbf{Q H}_{\text {ads }}$

$Q_{\text {ads }}+\mathbf{N A D H} \rightarrow \mathrm{QH}_{\text {ads }}+\mathrm{NAD}^{-}$

where $Q_{a d s} / Q_{\text {ads }}$ is the surface mediator redox couple. Such mechanism has been currently used to interpret the charge-transfer kinetics at chemically modified electrodes [32]. Some consequences arising from this type of mechanism for rotating disc electrode voltammetry have been analyzed [33].

Under stationary conditions, the surface concentrations $\Gamma_{Q}$ and $\Gamma_{Q H}$ of $Q$ and QH, respectively, do not vary with time, i.e.,

$\partial \Gamma_{\mathrm{Q}} / \partial t=k_{\mathrm{ox}} \Gamma_{\mathrm{QH}}-k_{\mathrm{ox}} P^{-i} \Gamma_{\mathrm{Q}}-k_{\mathrm{c}} \Gamma_{\mathrm{Q}} c_{\mathrm{NADH}}^{*}=0$

where $k_{\mathrm{ox}}$ is the potential-dependent anodic rate constant, $k_{\mathrm{c}}$ is the rate constant of the chemical step (reaction (IX)), $c_{\text {NADH }}^{*}$ is the NADH concentration at the electrode surface and $\boldsymbol{P}$ is the potential-dependent function

$P=\exp \left[(F / R T) \cdot\left(E-E^{\theta}\right)\right]$

Since the electrolyte solution is buffered, the proton concentration $c_{H^{+}}^{0}$ may be assumed to be constant throughout the solution space; it is included in the formal potential $E^{*}$ for electrochemical step (VIII),

$E^{0}=E^{0}+(R T / F) \ln c_{\mathbf{H}^{*}}^{0}$

In eqn. (1), the principle of detailed balancing [34] is used to interrelate the cathodic $\left(k_{\text {red }}\right)$ and anodic $\left(k_{\mathrm{ox}}\right)$ rate constants, $k_{\text {red }}=k_{\text {ox }} P^{-1}$.

Taking into account the fast follow-up oxidation of NAD' to NAD*, the diffusion current $I$ is given by

$I=(2 F A D / \delta)\left(c_{\text {NADH }}^{0}-c_{\text {NADH }}^{*}\right)$

where $A$ is the interfacial area, $D$ is the diffusion coefficient of NADH, $c_{\text {NADH }}^{0}$ is the 
bulk NADH concentration and $\delta$ is the thickness of the diffusion layer,

$8=1.6 i \omega^{-1,2} p^{1 / 6} D^{1 / 3}$

where $\omega$ is the angular velocity and $\boldsymbol{y}$ is the kinematic viscosity.

Assuming that

$\Gamma_{Q}+\Gamma_{Q H}=\Gamma_{0}=$ constant

a quadratic equation can be derived for the surface concentration $\Gamma_{Q}$ :

$P^{2}+P\left[s_{c}+s_{0 x} P(1+P)^{-1}-P(1+P)^{-1}\right]-s_{c} P(1+P)^{-1}=0$

where $p=\mathbf{r}_{Q} / \mathbf{r}_{0}$ and the dimensionless kinetic parameters $s_{c}$ and $s_{a x}$ are defined as

$s_{c}=D / k_{c} \delta \Gamma_{0}$

$s_{\text {ox }}=D c_{\mathrm{NADH}}^{0} / k_{\text {ox }} \delta \Gamma_{0}$

Finally, the current $I$ is given by

$I=p I_{d}\left(s_{c}+p\right)^{-1}$

where the limiting diffusion current $I_{\mathrm{d}}$ is

$I_{\mathrm{d}}=(2 F A D, \delta) c_{\mathrm{NADH}}^{0}$

Two limits of eqn. (7) can be distinguished. First, when the surface charge urausfer is very fast so that $k_{\text {ox }} \rightarrow \infty$ or $s_{\text {ox }} \rightarrow 0$ (the reversible case), eqn. (7) becomes [33]

$P=P(1+P)^{-1}$

In this case, the limiting current $i_{1}$ is given by

$I_{1}=I_{d}\left(1+s_{c}\right)^{-1}$

and the half-wave potential, $E_{1 / 2}^{r e r}$, is given by

$E_{1 / 2}^{r e o}=E^{\circ}+(R T / F) \ln s_{c}\left(1+s_{c}\right)^{-1}$

On the other hand, when the surface charge transfer is very slow (the irreversible case), quadratic eqn. (7) remains to be solved. However, due to the kinetic overporential, $P(1+P)^{-1} \rightarrow 1$ and

$p^{2}+p\left(s_{c}+s_{\alpha x}-1\right)-s_{c}=0$

The limiting current is again given by eqn. (13), but the half-wave potential depends on the kinetis parameters of the electrochemical step (VIII). If, formally,

$k_{\alpha o}(E)=k_{\mathrm{app}}^{0} \exp \left[(1-a) F\left(E-E^{0}\right) / R T\right]$

where $k_{\text {app }}^{0}$ is the apparent rate constant and $\alpha$ is the apparent cathodic charge transfer coefficient, the half-wave potential is given by

$E_{1 / 2}^{i \sin }=E^{0}+\frac{R T}{(1-\alpha) F} \ln \left[\frac{D c_{N A D H}^{0}}{k_{\text {ap }}^{0} 3 \Gamma_{0}}-\frac{2 s_{c}+1}{2\left(s_{c}+1\right)^{2}}\right]$ 
On the basis of this theory, we can make the following generalizations about the electrochemical oxidation of NADH at solid electrodes.

(1) Because the limiting current $I_{1}$ for the NADH wave is proportional to bulk concentration and $\omega^{1 / 2}$, and independent of $\mathrm{pH}$ and electrode material, we can conclude that it is controlled solely by diffusion of NADH to the electrode surface. Recalling eqns. (13 and 8), this means that $D / k_{c} \delta \Gamma_{0} \ll 1$. Using then this relation and eqn. (5) for $\delta$, a NADH diffusion coefficient of $3.3 \times 10^{-6} \mathrm{~cm}^{2} \mathrm{~s}^{-1}$ is calculated from the straight line slope in Fig. 6, which value is comparable to that calculated for NAD+ from its DME reduction (3.4 or 4.3) $\times 10^{-6} \mathrm{~cm}^{2} \mathrm{~s}^{-1}$ [2].

(2) As is apparent from eqns. (14 and 17), the nature of the electrode material may be reflected in the half-wave potential of the NADH wave through the formal potential $E^{\circ}$ of the surface redox couple (reaction (VIII)). In fact, the differences in $E_{1 / 2}$ qualitatively correlate with the differences in onset of oxygen adsorption on comparing the waves both at different electrodes (Figs. 2 and 4) and at different $\mathrm{pH}$ at the same electrode (Fig. 2, (Au); Table 2). However, the shift of $E_{1 / 2}$ with electrode material is considerably less than with onset of oxygen adsorption, e.g., in going from Au to GC, $E_{1 / 2}$ changes by ca. $0.6 \mathrm{~V}$ (Fig. 4), while the start of oxygen adsorption changes by ca. $1.1 \mathrm{~V}$ (Fig. 2). This can be ascribed to other factors, e.g. to the change in $k_{\text {app }}^{0}$ or $\Gamma_{0}$ (cf. eqn. (17)).

(3) The surface redox couples which are presumably involved in NADH oxidation at solid electrodes exhibit grossly irreversible electrochemical behavior, although, for example, the oxygen adsorption at Pt $[21,23]$ or Au $[21,22]$ electrodes is probably initiated by the reversible adsorption of $\mathrm{OH}$ species. Consequently, the irreversible NADH oxidation at solid electrodes can be expected. In fact, the reciprocal slopes of the logarithmic analysis of the anodic NADH wave (Table 2) are much higher than the reversible slope of $0.059 \mathrm{~V}$, which is predicted by the equation

$$
\left(I_{1}-I\right) I^{-1}=s_{c}\left(1+s_{c}\right)^{-1} P^{-1}
$$

derived for the reversible case from eqns. (10, 12 and 13$)$.

However, in the irreversible case, the non-linear logarithmic analysis can be expected, due to quadratic eqn. (15). At the foot of the irreversible wave, $p \ll 1$ and $\left(I_{1}-I\right) I^{-1}-s_{\text {oux }}$, i.e, the reciprocal slope of the logarithmic analysis approaches 2.303RT/(1-a)F. The non-linear logarithmic analysis is observed for NADH oxidation at all three electrodes, but its reciprocal slopes do not vary too much from the values given in Table 2, which would correspond to an apparent charge-transfer coefficient $\alpha$ of about 0.7 .

(4) In general, the half-wave potential of the NADH anodic wave may depend on the electrode rotation speed, NADH concentration and solution pH (cf. eqns. (14 and 17)). In the reversible case (eqn. (14)), only the effect of the solution pH should be observed, $\Delta E_{\mathrm{i} / 2}^{\mathrm{rev}} / \Delta \mathrm{pH}=-2.303 R T / F$, while $\left(\Delta E_{\mathrm{i} / 2}^{\mathrm{rev}} / \Delta \log c_{\mathrm{NADH}}^{\mathrm{o}}\right)=$ $\Delta E_{1 / 2}^{\mathrm{rev}} / \Delta \log \omega=0$. On the other hand, for the irreversible case, $\Delta E_{1 / 2}^{\mathrm{irr}} / \Delta \mathrm{pH}=0$, while $\Delta E_{\mathrm{rev}}^{\mathrm{irr}} / \Delta \log \omega=2.303 R T / 2 F(1-\alpha)$ and $\Delta E_{\mathrm{i} / 2}^{\mathrm{ir}} / \Delta \log c_{\mathrm{N} A \mathrm{DH}}^{0}=2.303 R T / F(1$ $-\boldsymbol{a})$.

If $\alpha$ is assumed to be 0.7 , the effects observed on NADH oxidation at $\mathrm{Pt}$ and $\mathrm{Au}$ 
electrodes point to the quasi-reversible behavior of the surface redox couple, with the exception of the effect of the electrode rotation speed which corresponds rather to the tehavior of an irreversible mediator redox system.

\section{ACKNOWLEDGMENT}

The authors thank the National Science Foundation which helped support the work described.

\section{REFERENCES}

1 A.L. Underwood and R.W. Burnet in A. Bard (Ed), Electroanalytical Chemistry, Vol. 5. Marcel Delker, New York, 1972 Ch. 1.

2 F.J. Eving, CO. Schmakel and K.S.V. Santhanam, Crit. Revs. Anal. Chem., 6 (1976) 1.

3 PJ. Elving in G. Milkzzo (Ed), Topics in Bioejectrochemistry and Bioenergetics, Wiley-Interscience, 1976, Vol L. P. 278.

4 H. Jeoffddi, J. Eloctroanal Chem, 110 (1980) 295.

S P.J. Elving W.T. Bresnahan, J. Moirowx and Z Sames, Bioclectrochem. Bioenerg. 7 (1980) 125.

6 J.N. Barnett and A.L. Undermood, Biochemistry. 4 (1965) 2060.

7 R.D. Bram K.SV. Senthannm and PJ. Eving J. Am. Chem. Soc, 97 (1975) 2591.

8 W. Brodel and R.A. Jenkins, Anal. Chem. 47 (1975) 1337.

9 H. Inegfeldh, A. Torstensson and G. Johansson, Anal. Chim Acta 97 (1978) 22

10 M. Aizzwa, RN. Coughlin and M. Charies, Biochem. Biophys. Acta. 385 (1975) 362

11 W. I. Bhodel and R.G. Hans, Acal Chem 42 (1970) 918.

12 J. Moiroux and P.J. Eving, J. Am. Chem. Soc, 102 (1980) 6533.

13 MA Jensen, PhD. Thesis, University of Michigan, Ann Arbor, 1977.

14 I. Moiroux and P.J. Elving Anal Chem. 50 (1978) 1056.

15 J. Moiroux and PJ. Eking Anal Chem, 51 (1979) 346.

16 J. Moiroux and P.J. Elving J. Electroanal Chem, 102 (1979) 93.

17 D.C. Tse and T. Kuwrana, Anal. Chem. 50 (1978) 1056.

18 It. Jeegfeldh. A. A.C. Torstensson. LG.O. Gonon and G. Johansson. Anal Chem. 53 (1981) 1979.

19 D.A. Hall and P.J. Eving Anal. Chim Acta, 39 (1967) 141.

20 D. Jain and W. Vielstich, I. Electrochem. Soc. 109 (1962) 849

21 R. Woods in A.S. Bard (Ed), Electroanalytical Chemistry. Vol 9. Marcel Dekker, New York, 1976, P. 1.

22 M. Sotra. J. Eloctroenal Chem 72 (1976) 287.

23 B. . Thak B.E Conway and H. Angerstein-Koslowska, J. Electroanal Chem. 48 (1973) 1.

24 W. Bold and M Breiter. Bectrochim Acta, 9 (1964) i025.

25 H. Angerstein-Koelowsia and BE Conway. J. Electroanal Chem, 95 (1979) 1.

26 A. Michri, A.G. Pshchenichnikov and R.Kh Eurshtein, Soviet Electrochem. 8 (1972) 351.

27 T. Biegler. DA.T. Rand and R. Woods, J. Electroanal. Chem. 29 (1971) 269.

28 DAJ. Rand and R. Wrods, J. Electroanal Chem. 35 (1972) 209.

29 K. Taltanmun, A. Mori and F. Kussu, Biocketrochem. Bioenerg. 8 (1981) 229.

30 CPK Precision Molecular Models, The Ealing Corp 2225 Massachusetts Ave. Cambridge. MA. 02140, U.SA.

31 Z Samoe and J. Weber, Eectrochim. Acta, 20 (1975) 403.

32 R.W. Murray, Aoc Chem Rea, 13 (1980) 135.

33 C.P. Andriext, J.M. Dumas-Bouchint and J.M. Saveant, J. Electroanal. Chem. 123 (1981) 171

34 R.R. Dogonadies in N.S. Hush (Ed), Reactions of Molocules at Electrodes Wiley-Intersciencr, New: York, 1971. p. 135. 